\title{
Electron Emission from Ferroelectric/Antiferroelectric Cathodes Excited by Short High-Voltage Pulses
}

G. Benedek*, I. Boscolo*, J. Handerek**, H. Riege***

\begin{abstract}
Un-prepoled Lead Zirconate Titanate Lanthanum doped-PLZT ferroelectric cathodes have emitted intense current pulses under the action of a high voltage pulse of typically $8 \mathrm{kV} / \mathrm{cm}$ for PLZT of $8 / 65 / 35$ composition and $25 \mathrm{kV} / \mathrm{cm}$ for PLZT of $4 / 95 / 5$ composition. In the experiments described in this paper, the exciting electric field applied to the sample is directed from the rear surface towards the emitting surface. The resulting emission is due to an initial field emission from the metal of the grid deposited over the emitting surface with the consequent plasma formation and the switching of ferroelectric domains. These electrons may be emitted directly from the crystal or from the plasma. This emission requires the material in ferroelectric phase. In fact, PLZT cathodes of the $8 / 65 / 35$ type, that is with high Titanium content, showing ferroelectric-paraelectric phase sequence, emit at room temperature, while PLZT cathodes of the 4/95/5 type, that is with low Titanium content, having antiferro-ferro-paraelectric phase sequence, emit strongly at a temperature higher than $130^{\circ} \mathrm{C}$.
\end{abstract}

* University, INFN and INFM, Milan, Italy

** University of Silesia, Katowice, Poland

***CERN, LHC Division

Journal of Applied Physics

Administrative Secretariat

Geneva, Switzerland

LHC Division

24 November 1997

CERN

CH - 1211 Geneva 23 


\section{1- Introduction}

Strong electron emission from ferroelectric (FE) materials, stimulated either by short electric field pulses (field direction from the emitting surface of the cathode towards the rear surface) or by laser light pulses or by a combined electrical-optical stimulation has been observed and studied since 1987 [1-6] at CERN. Emission with ferroelectric cathodes but with opposite direction of the exciting field is presently under investigation in many laboratories [7-13]. Also in this paper we deal with this latter mode of excitation.

The cathodes are shaped in form of disks. The

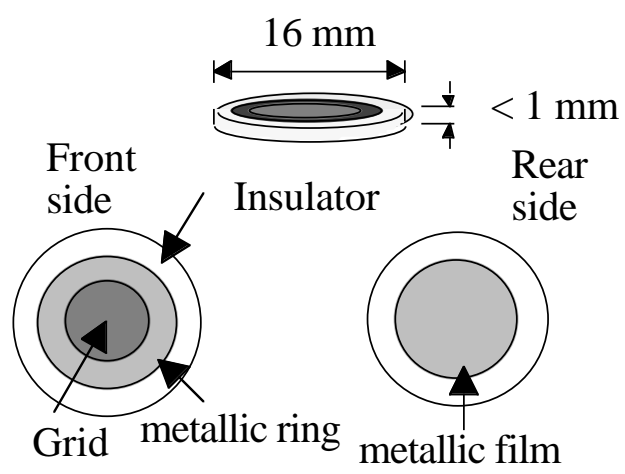

Fig. 1. Skethch of the PLZT disk used as cathode.

[the positive end $\{+\}$ towards the orid electrode], the excitation of the sample is normally made by applying a negative HV pulse on the rear electrode [1-3].

The prepoling process creates, in addition to the spontaneous polarization $\mathrm{P}_{\mathrm{s}}$, a space charge polarization $\mathrm{P}_{\mathrm{SC}}$, due to the induced migration of defects (oxygen and lead vacancies) to the external surfaces. This fact makes the system more complex, but does not change the simple phenomenological model of the emission mechanism.

In this work we have studied, instead, the emission from crystals without prepoling. We have tested the behaviour for either a positive excitation to the rear surface or a negative excitation to the front surface. It has been used a reverse excitation field with respect to experiments in refs [1] to [3].

The ferroelectric materials under investigation are made from Zirconium-rich LeadLanthanum-Zirconium-Titanate PLZT x/y/1-y, of composition $\left[\mathrm{Pb}_{1-\mathrm{x}}, \mathrm{La}_{\mathrm{x}}\right]\left[\mathrm{Zr}_{\mathrm{y}}, \mathrm{Ti}_{1-\mathrm{y}}\right] \mathrm{O}_{3}$, where $\mathrm{x}$ and $\mathrm{y}$ are the fractional compositions of $\mathrm{La}$ and $\mathrm{Zr}$. This Lead Zirconate Titanate Lanthanum doped material is called in abbreviation PLZT. Different PLZT samples, characterized by different compositions, and/or different levels of polarization, lead to different behaviours. We classify PLZT materials in two families depending on Zirconium/Titanium ratio: the first one is PLZT-x/95/5, with a low Titanium content; the second one is PLZT-x/65/35, with a high Titanium content. The two kinds of material have a different phase state at room temperature, as shown in fig. 2. PLZT with a $\mathrm{Zr} / \mathrm{Ti}$ ratio close to $65 / 35$ and Lanthanum content less than $10 \%$ is in ferroelectric (FE) phase state, while PLZT with a $\mathrm{Zr} / \mathrm{Ti}$ ratio close to $95 / 5$ is in antiferroelectric (AFE) phase state, provided $L a$ concentration is not too large [10-14].

However, the application of an electric pulse induces an antiferro-ferroelectric transition in a 95/5 crystal, while induces only a variation of the polarization state in a 65/35 crystal. The hysteresis loop in the two cases is very much different, as shown in fig. 2, so the application of an electric field entails in the two cases a different level of polarization.

We chose for our investigation the two ceramic materials PLZT-8/65/35 and 4/95/54/94/6-2/94/96. The former is explicitly chosen because it is close to the phase boundary between the tetragonal and rhombohedral ferroelectric phases and to the region of the socalled pseudo-ferroeletricity (SFE dashed area, see fig. 2). The material pertaining to that SFE zone, has the characteristics of having a diffuse phase transition and a relaxor behaviour after an excitation. In the case of the family 95/5, the crystal undergoes an 
antiferro-ferroelectric phase transition around $120^{\circ} \mathrm{C}$ (this temperature is shifted downwards when an electric field is applied [1,16]). Furthermore, starting from that temperature, there is the coexistence of ferroelectric and antiferroelectric phases, which leads to a diffuse phase transition. These features are originated by the differences in local phase transition temperature, differences that in turn come from local composition fluctuation and local electric field fluctuation and anisotropic strains in the bulk.

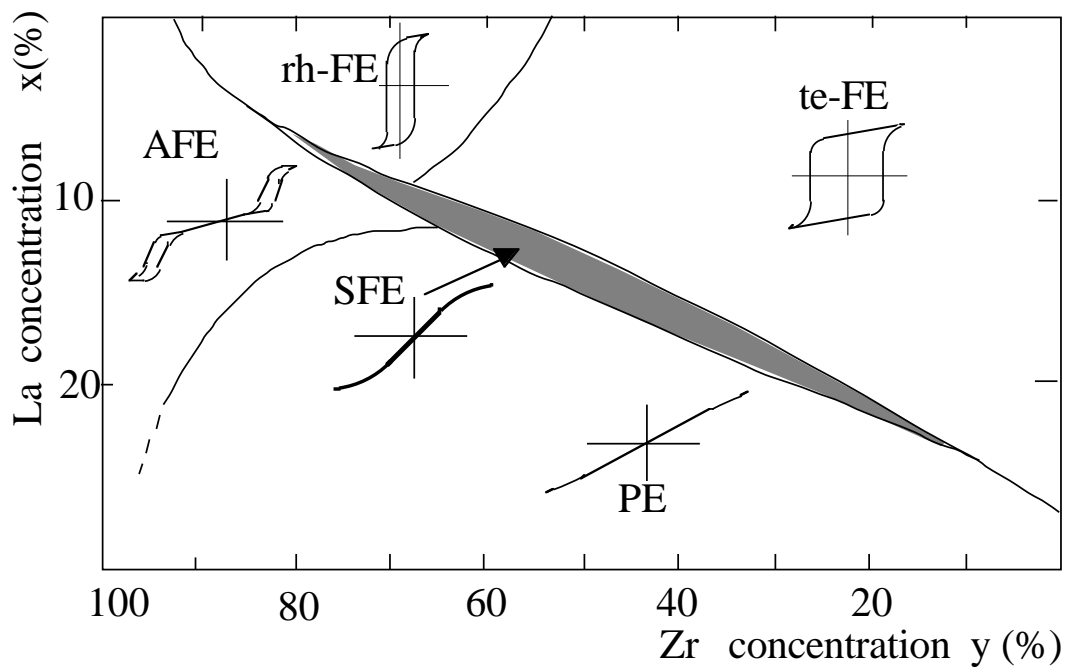

Fig. 2. Room temperature phase diagram for PLZT materials showing ferroelectric (FE), antiferroelectric (AFE) and paraelectric (PE) phases as function of composition. The pseudo-ferroelectric (SFE) dashed region corresponds to a diffuse, metastable ferroelectric phase, that exhibits a gradual change of physical properties [from ref.15].

\section{2- The phenomenological picture of the emission mechanism}

The electrically stimulated electron emission mechanism is a bit different in the two cases of prepoled and un-prepoled crystal. When a negative high voltage pulse is applied to a prepoled PLZT sample against the spontaneous polarization, the latter is suddenly lowered (due to change in orientation of $\mathrm{P}_{\mathrm{S}}$ vectors in the individual domains), or even completely reversed, and the space charge free electrons are pushed out from the crystal surface into the vacuum gap and here they are accelerated. This emission of space-charge

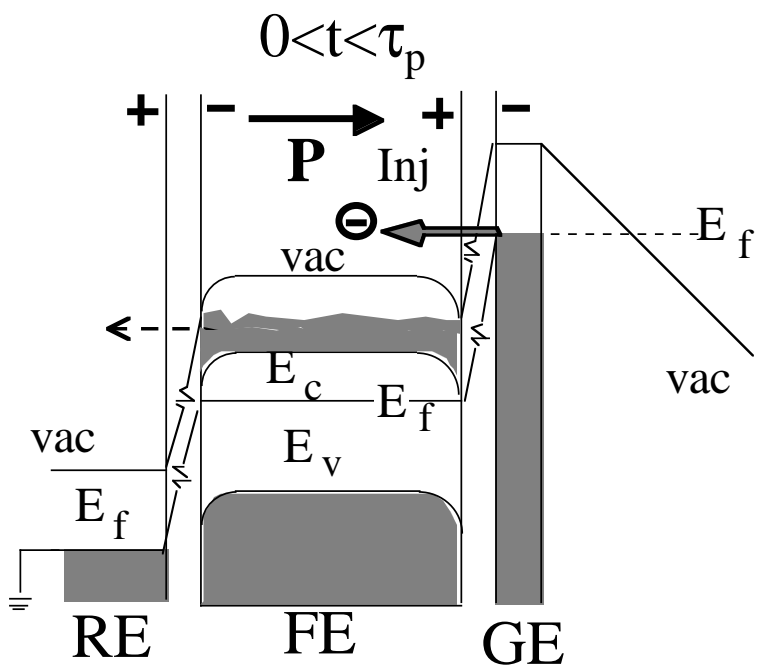

Fig.3. Sketch of the band bending owing to the application of negative voltage pulse to GE electrode: Inj are electrons injected from the metal into the crystal surface region; vac is the crystal vacuum level, $\mathrm{E}_{\mathrm{f}}, \mathrm{E}_{\mathrm{c}}$ and $\mathrm{E}_{\mathrm{V}}$ are respectively the Fermi, the conduction bottom and the valence top levels. electrons, due to the sudden lowering of the vacuum barrier and the mutual coulomb repulsion, has been called ferroelectric emission or self-emission. One of the main features of this emission is that the emitted electron can be highly energetic, some electrons acquiring a kinetic energy up to twice or more than the pulse voltage due to Auger processes.

We discuss in more details the emission process when the sample is unprepoled. A different mechanism occurs in this case. We consider the case of a negative voltage pulse of duration $\tau_{\mathrm{p}}$ applied to the front electrode (equivalent to applying a positive pulse to the rear electrode from the point of view of the electric field within the crystal). The leading edge pulse sets a polarization with the positive end $[+]$ towards the grid, i.e. a layer of a positive bound charge sets at the interface of the cathode with the grid stripes (see fig.3). This charge is 
compensated in two different ways in metallic and bare parts of front surface. In the part covered by the metal, the positive charge is compensated both by negative charges induced on the internal surface of the metal and by the electron charge injected into the shallow traps in the surface of the crystal. On the bare strips the positive charge is compensated by electrons emitted by the borders of the metallic strips (this emission is caused by the high field strength) and then migrated over that bare part. Part of electrons enter the surface traps and part may form an electron cloud. The internal and external space charge is the charge ready for the emission. In fact, when the polarizing electric field is switched off and so in turn $\mathrm{P}_{\mathrm{S}}$ polarization relaxes back (the material is a relaxor, the domains change their orientation and some of them reverse), the positive charge lowers, thus the negative counterpart charge remains in excess. The negative carriers within the metallic part go away through the circuit, while the carriers in the bare strips may go either back into the metallic strips, or to vacuum. In case of polarization reversal, the crystal surface switches from positive to negative, thus both trapped and external electrons are ejected into the vacuum (see fig. 4). The change of polarization is extremely fast in PLZT relaxor. From previous model, it comes out that the cycle polarizationcharge injection-depolarization-charge ejection is repeatable.

Because of the dramatic band bending induced by polarization, a free-electron space charge rapidly $\left(\tau_{\mathrm{d}} \approx\right.$ few $\left.n s\right)$ forms around the stripes below the interface and at the regions of the free ferroelectric surface surrounding the strips. The barrier towards vacuum for free electrons in such region is considerable lower than the ordinary fieldemission barrier of the metal grid. Thus ferroelectricity provides, through its pulsed induced polarization, a short circuit channel for field emission.

The emission of electrons from a crystal into the vacuum requires a strong bending of bands at the surface, the Fermi level is positioned nearer to the conduction band (see fig. 3), so electrons can either tunnel out pushed by the repulsing potential, or exit the crystal because of the Auger process. From fig. 3 to fig. 5 the emission mechanism is explained in terms of bands changement for the application of the voltage pulse to the PLZT disc.

We want to stress that the theoretical foundation of the model we are going to present is valid for a single crystal in the monodomain state (with $\mathrm{P}_{\mathrm{S}}$ homogeneous). Instead, being PLZT a ceramic material, it consists of small crystal grains separated by glass like boundaries (morphotropic phase) and it is at certain extent a porous material. In addition, there are electron-hole and mobile ion space charge which affect the domain dynamics within the grains. For this reason only part of the domains have reversible spontaneous polarization. However, since ceramics have a behaviour very much similar to large homogeneous single crystals, the model should be applicable to them. In fact, it ought to hold for those grains and domains which participate in the variation of $\mathrm{P}_{\mathrm{S}}$ caused by the external field (and consequently in the relevant electron emission), and a polydomain structure behaves like a monodomain one as soon as domains get oriented.

In the unpolarized case there is already a surface band bending due to existence of surface electronic states (where the surface is free), or to the matching of Fermi levels with the metallic electrodes at equilibrium (where the surface is covered by the metallic film). In this case (our case) the band bending is upward (as in these figures) if work function of metal $\left(\varphi_{\mathrm{M}}\right)$ is bigger than the work function of ferroelectric $\left(\varphi_{\mathrm{E}}\right)$, but downward if $\varphi_{E}>\varphi_{M}$. We have assumed the former case. This is an important point to have in mind, because it has consequences on the intensity of secondary or inverse ejections. In our representation the Fermi level of ferroelectric has been moved towards the conduction bottom level $\left(\varepsilon_{\left.\mathrm{F} \sim \varepsilon_{\mathrm{C}}\right)}\right.$ because the material is heavily doped.

As sketched in fig. 3, soon after a negative pulse on the grid electrode has started, the ferroelectric domains are oriented and the surface polarization charge screens at a good extent the pulse field. Electrons are injected from front electrode into the ferroelectric surface region well above the ferroelectric vacuum level and form, at the free (intergrid) ferroelectric surface, a plasma. A moderate ejection of electrons into vacuum can start now.

However, as soon as the pulse is switched off, space charge confinement is over, ejection becomes explosive (see fig. 4). For a short time interval just after the end of the pulse and before the space charge formation there is a situation of unequilibrated bands as shown in 
fig. 4a. Note that the surface band bending at front surface and rear surface are not equal in size because they include the original small symmetric band bending of the unpolarized state. The space charge formation brings a large amount of electrons, in the short dielectric relaxation time $\tau_{\mathrm{d}}$, down in the low energy states of the space charge region near the front surface and energy has to be dissipated in some way. Because of such a high carrier density the dominant energy dissipation mechanism is the Auger process, in which other electrons, especially those of the hot plasma, get the energy through collisions and are ejected into vacuum.
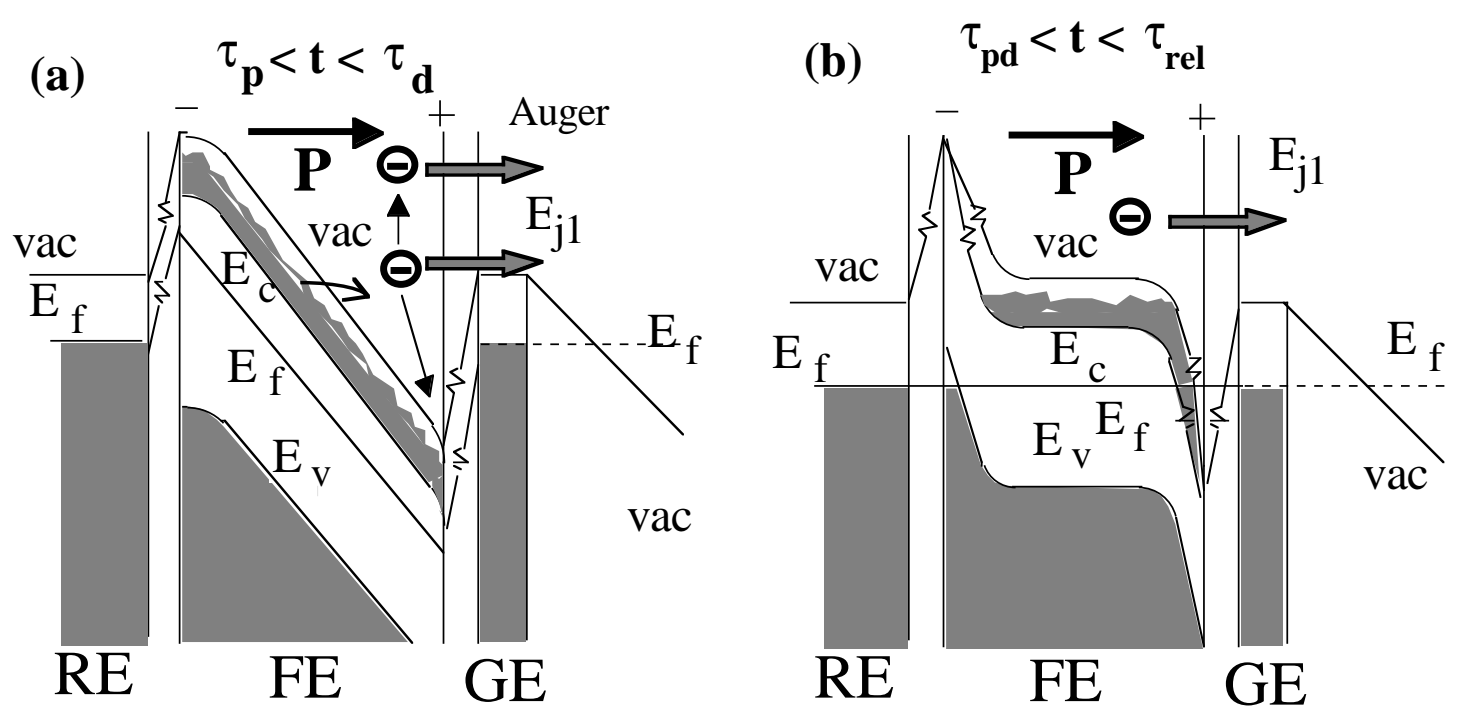

Fig. 4. Sketch of band bending when the exciting pulse is over: Ej represents electrons ejected from the crystal surface into the vacuum; the meaning of the symbols are as in previous figure and in the text; frame $4 \mathrm{a}$ refers to the transient band configuration which sets for a short time interval just after the exciting pulse; $\tau_{\mathrm{pd}}=\tau_{\mathrm{pulse}}$, relax .

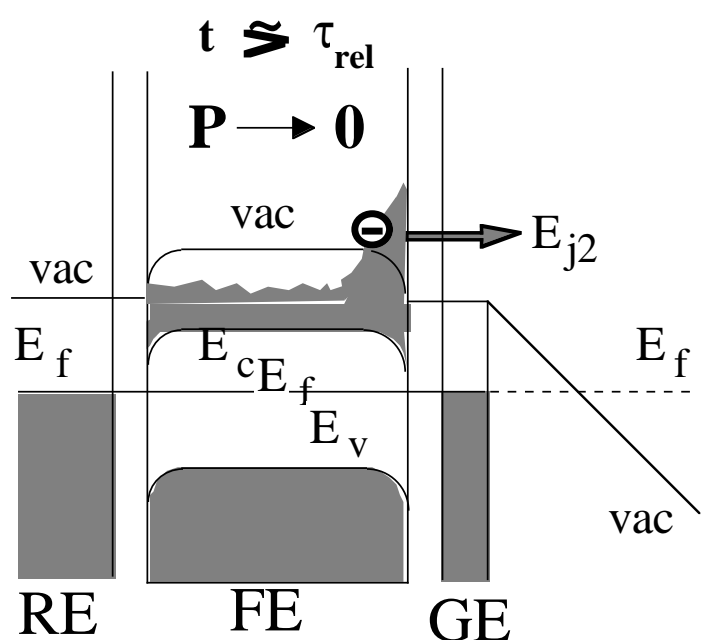

Fig. 5. Sketch of bands configuaration with the lifting up of the space charge due to the sudden disappearing of the polarization. Since in this case we have a second current peak we have named the ejected electrons $\mathrm{E}_{\mathrm{j} 2}$. macroscopic polarization fades off in a time $\tau_{\text {relax }}$ In after the end of the polarizing pulse and the accumulated space-charge is lifted up, as shown in fig. 5. If $\tau_{\text {relax }}>>\tau_{d}$ the disappearance of the space-charge is adiabatic and nothing happens as regards ejection. If energies can be much larger than the original pulse voltage. this phenomenon should be observed in the first stronger peak immediately following the pulse descending edge.

Since the surface polarization charge of the ferroelectric remains uncompensated, free carriers inside the ferroelectric rapidly (within the dielectric relaxation time $\tau_{\mathrm{d}}$ ) redistribute and form space charges of opposite sign at the front and rear surfaces. The space-charge depth is generally given by the Debye length $L_{D}=\left(k T / \varepsilon_{s} n\right.$ $\left.\mathrm{e}^{2}\right) 1 / 2$ where $\varepsilon_{\mathrm{s}}$ is the dielectric constant and $\mathrm{n}$ the carriers density. Here we have an n-type semiconductor and therefore free electrons accumulate at front surface, while the space charge at rear surface is mostly due to uncompensated ionized donors [2]. macroscopic polarization fades off in a time $\tau_{\text {relax }}$ In after the end of the polarizing pulse and

Due to Auger processes the ejection 
the two times are comparable or $\tau_{\text {relax }}<\tau_{\mathrm{d}}$, the rapid lowering of the polarization determines a second ejection pulse of those electrons which had been temporarily trapped during the recipient space charge formation. In this case, we have a second emission peak immediately following the stronger first one, which is composed by less energetic electrons (this is what we have observed, see below). The time separation measures the relaxor $\tau_{\text {relax }}$.

We note that during previously described processes free charge motion occurs also on the rear side in a way easily argued from the band diagrams. At the stage of figure 4, electrons can be injected from rear electrode into ferroelectric which may produce a delayed ejection pulse at front surface.

We point out that in the configuration adopted in this work, negative high voltage pulsing at the grid electrode, the injection of electrons in the intergrid surface region (fig. 3), leads to plasma formation. The surface field due to the charge unbalance between the bound and free charge on the front surface of the dielectric becomes so high (of the order of some $\mathrm{MV} / \mathrm{m}$ ) that the metallic stripes flushes electrons and the metallic rims are expected to form a plasma which will cover the emitting area. In this emission a low external voltage is able to provide large current: the diode impedance is low owing to the presence of plasma.

The physics behind the emission in this regime is complicate: the crystal dynamics for the pure electron emission is determined by many elementary processes inside the crystal, but also depends on the dynamics of the surface charge with the charge within the diode gap [17]. We may expect that the interpretation so far proposed is far from being completely exhaustive. With the experiments presented in this article and the relevant interpretations, we hope to give a further contribution to the understanding of the physics (the role of individual factors, various interactions, inferences between these, etc.) lying behind the emission from ferroelectric ceramics.

In the case of ferroelectric field assisted emission, the cathode emits a much larger amount of charge.

\section{3- THE EXPERIMENTAL APPARATUS}

A- The sample preparation. The investigated PLZT ceramics were prepared using the conventional mixed oxide method. The thermal synthesis of blended and pressed $\mathrm{PbO}$, $\mathrm{ZrO}_{2}, \mathrm{TiO}_{2}$ and $\mathrm{La}_{2} \mathrm{O}_{3}$ oxides was carried out at $950^{\circ} \mathrm{C}$ for $3 \mathrm{~h}$. The milled and coldpressed cylinders were then sintered at $1100{ }^{\circ} \mathrm{C}$ and finally at $1250{ }^{\circ} \mathrm{C}$ each for $3 \mathrm{~h}$. These sintering processes were carried out in a double crucibles with a $\mathrm{PbO}$ atmosphere to maintain the established composition. The cathodes are shaped in form of disks of thickness less than $1 \mathrm{~mm}$ and diameter of about $16 \mathrm{~mm}$. The disk is polished and then coated with a thin metallic film of uniform thickness on the rear surface and in the form of a grid on the front front surface (Fig.1). The metallic stripes, $200 \mu \mathrm{m}$ wide and separated by $200 \mu \mathrm{m}$ gaps, are connected by a conductive ring. The outer bare edge of the sample is covered by an insulating layer. The bare surface, which is emitting, is approximately $\mathrm{A} \approx 0.3 \mathrm{~cm}^{2}$.

B- Experimental set-up. The tests have been done with the experimental setup shown in Fig.6. The diode electrodes have the Pierce design in order to have a uniform accelerating field within the diode gap $[18,19]$. The ferroelectric disk is hold in place and electrically connected to the pulser by the rim of the Pierce electrode which is gently pressed onto the metallic ring connecting the grid stripes. The sample is mounted on an electrically insulating support. The connection of rear and front electrodes to the high voltage pulser is designed so to avoid deformations on the rectangular high power pulse. A resistance of $100 \Omega$ is set in parallel to the capacitance of the disk for matching purpose. The temperature of the ferroelectric sample can be raised by means of a heater set on the back of the sample and is measured with a thermocouple. When an accelerating voltage $V_{\text {acc }}$ is applied, a positive high voltage generator is connected to the anode plate through the holding stem, which is then connected to the diagnostic circuit by a capacitor. The current and the charge are monitored by a voltage divider and an $\mathrm{RC}$ integrator circuit. 

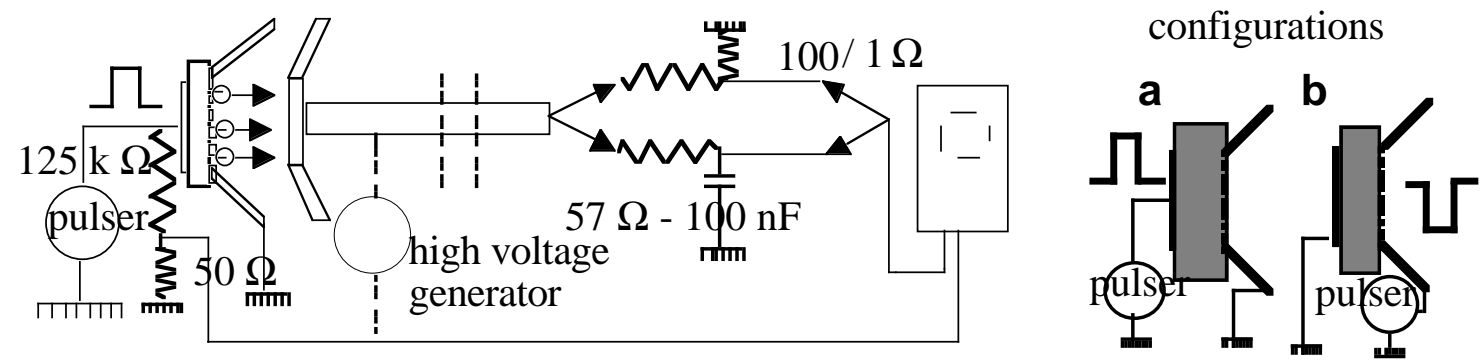

Fig. 6. Sketch of the setup for the charge and current measurement; the exciting pulse can be either negative (negative excitation) or positive (positive excitation). The cathode is inserted in a Pierce designed diode. A decoupling capacitor is inserted when an accelerating voltage is applied, otherwise the stem is electrically connected to ground. On the right, the electrical connections for the two configurations a and b.are sketched.

The measurements have been done in the following two different configurations:

(a) a positive pulse is applied to the rear electrode (the front electrode is hold at ground); (b) a negative pulse is applied to the front electrode (the rear electrode is hold at ground).

In the first case an accelerating voltage $V_{\text {acc }}$ is applied through the diode gap with the aim of investigating the variation of the emitted current with increasing $\mathrm{V}_{\mathrm{acc}}$. In the second case the negative $\mathrm{HV}$ pulse at the front electrode, besides changing the polarization $\mathrm{P}_{\mathrm{S}}$ of the crystal, also sets an accelerating voltage, so that no extra $\mathrm{V}_{\text {acc }}$ is needed.

The measured quantities are: (1) the switching pulse before entering the flange where is fixed the system holding the sample, (2) the current arriving at the collector by a 100/1 voltage divider and (3) the charge by an RC integrator $57 \Omega-100 \mathrm{nF}$. The waveforms have been recorded by a storage oscilloscope with a sampling of $5 \mathrm{GHz}$. Often a $30 \mathrm{MHz}$ bandwidth limitation had to be inserted in order to eliminate noise.

The amplitude of the electromagnetic noise is of the order of $2 \mathrm{mV}$ at both the integrator and the current divider, with a switching HV pulse of about $2 \mathrm{kV}$ applied to the rear electrode. When the HV pulse is applied to the front electrode, we get an electrical disturbance of about $150 \mathrm{mV}$, because the HV pulse is capacitively coupled to the measuring system. When the pulse is applied to the rear electrode, the HV pulse is screened by the grid and the connected Pierce electrode, which are kept at ground potential.

\section{4- EXPERIMENTAL RESULTS WITH DISCUSSION}

\section{1- Sample PLZT - 8/65/35 with a thickness of $1 \mathrm{~mm}$}

(a)- Positive pulse on rear electrode

The emission regime with rear electrode positively pulsed has been experienced in other labs but with different ferroelectric materials [17], while the regime for negative front surface has not been studied before. The two cases show somewhat different behaviours because the emission depends greatly on the surface behaviour and the field distribution and the free electron dynamics in the vicinity of the emitting surface are quite different in the two cases.

We point out that in these configurations the metallic grid stripes are negative with respect to the rear electrode, so their rims may emit for field emission when the electric field is high enough (see fig. 7). The applied electric field is almost concentrated nearby the stripes because of the high value of the dielectric constant $(\varepsilon>300)$, so it is expected to have field emission from the metallic stripes and in turn the plasma formation nearby. This plasma expands over the whole bare ferroelectric area. The whole cathode ought to be covered by a plasma sheet after a while. 

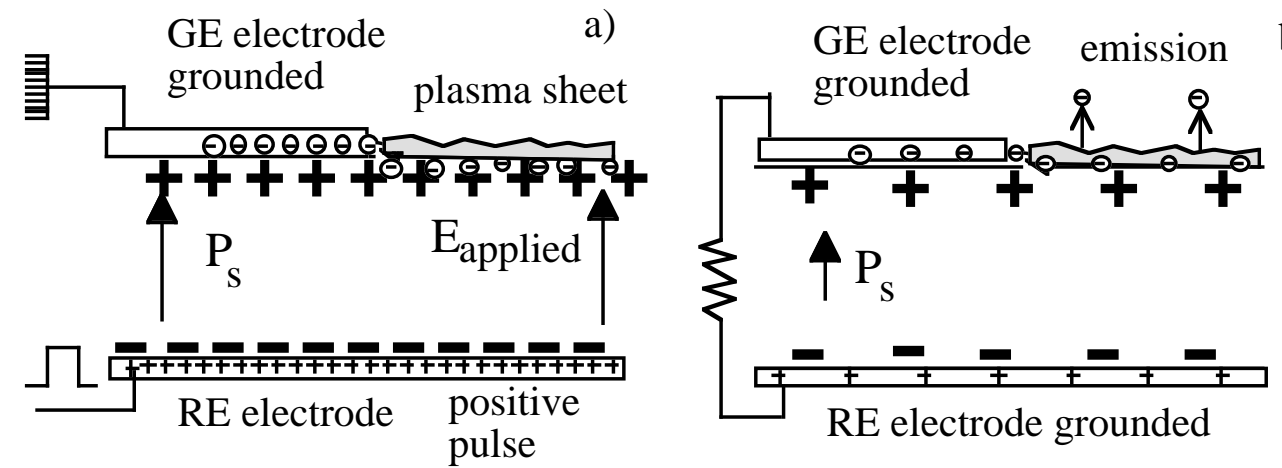

b)

Fig. 7. Sketch of the system; a) during the electric pulse: the unbalanced bound charges are compensated by free charges emitted by the metal grid during the electric pulse and expanded over the whole surface; b) after the switching of the pulse: the external field disappears, the polarization is reduced, some of the free charges are expelled.

Because of the electron cloud-plasma, if an accelerating voltage is applied, an intense current is obtained. Therefore very different current amplitudes are expected without and with an accelerating voltage applied trough the diode gap.

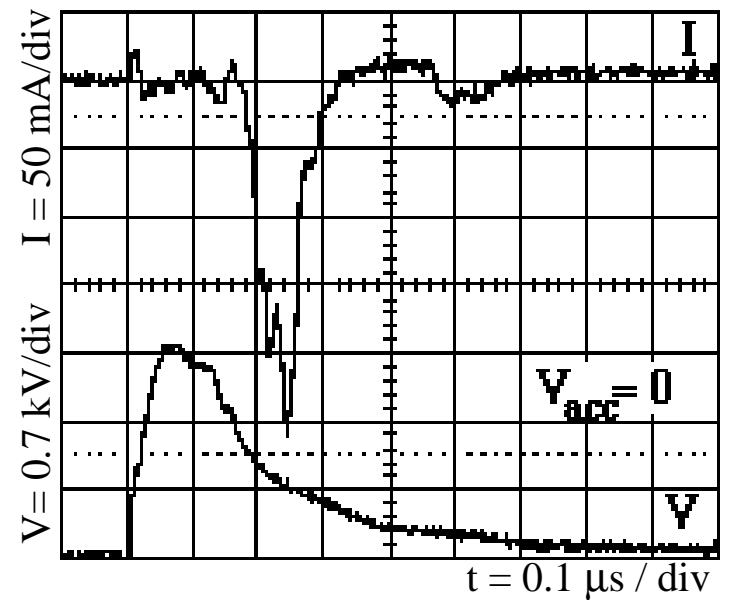

Fig. 8. Typical current waveform with a high voltage pulse $\mathrm{V}$ RE,HV $=2 \mathrm{kV}$ (bottom curve), $\mathrm{V}_{\mathrm{acc}}=0, \mathrm{I} \approx 300 \mathrm{~mA}$ (top curve). We remark that the rising and decaying times of the voltage pulse increases of about $20 \%$ connecting the ferroelectric capacitor with respect to open circuit.
The emitted current without accelerating voltage and an applied field strength of $20 \mathrm{kV} / \mathrm{cm}$ is shown in fig. 8 , together with the driving HV pulse.

The current pulse shows up constantly about $50 \mathrm{~ns}$ after the peak in the HV pulse. Its width is more often around $70 \mathrm{~ns}$, the peak value is about $300 \mathrm{~mA}$. We have measured the stability of the current and charge emission as function of the exciting $\mathrm{HV}$ pulse. At a voltage of $10 \mathrm{kV} / \mathrm{cm}$, there is a considerable pulse-topulse scatter in current amplitude and shape, while at a voltage amplitude greater than $20 \mathrm{kV} / \mathrm{cm}$ the stability reached a value of $90 \%$.

The current is collected at zero accelerating voltage. This means that the electrons are enough energetic to counter the space charge force, which, from simulations with E-GUN code, results in some $\mathrm{kV}$. The facts that the electrons are energetic and that they are emitted at the trailing edge of the exciting voltage pulse, drive to the conclusion that the emission is of ferroelectric type, that is the electrons are launched by the crystal into the vacuum with a considerable initial velocity. A further characteristic of the ferroelectric current pulse is the appearing of a second peak after $200 \mathrm{~ns}$. In fig 8 it is small, but in many other shots it was more pronounced. The intense current of energetic electrons and the two peaks in sequence match with the physical description of figs. 4 and 5. 


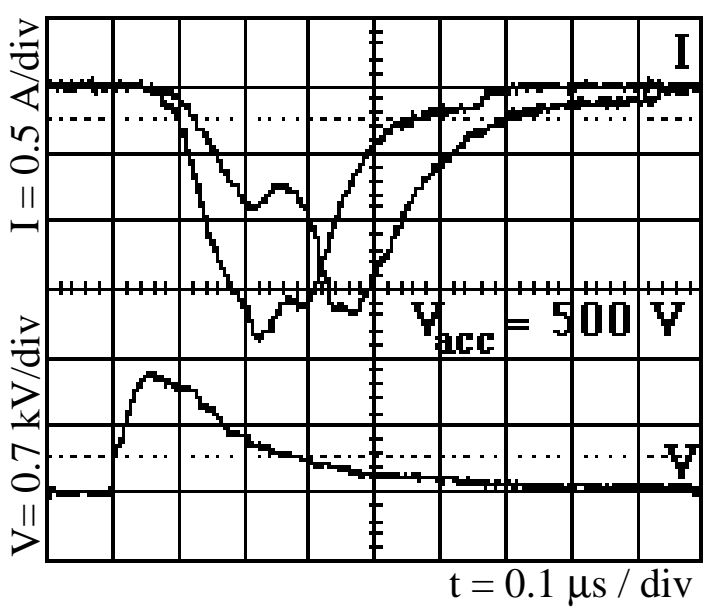

(a)

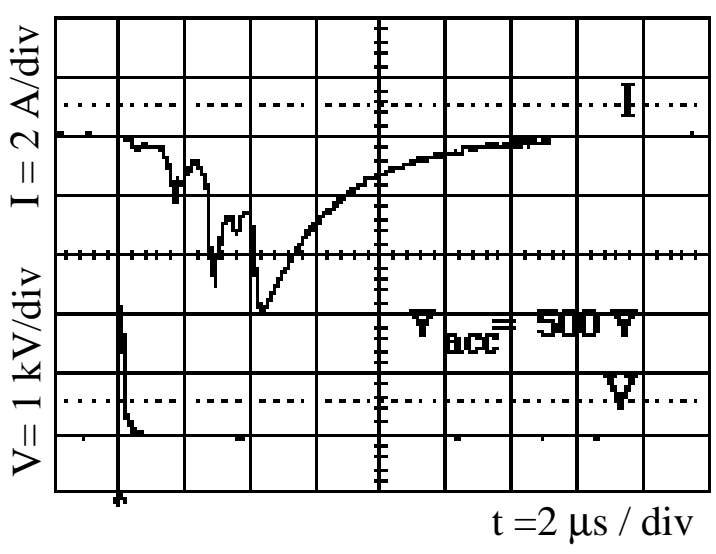

(b)

Fig. 9. Typical current wave-forms: upper frame $\mathrm{V}_{\mathrm{RE}, \mathrm{HV}}=1.2 \mathrm{kV}, \mathrm{V}_{\mathrm{acc}}=500 \mathrm{~V}, \mathrm{I}_{\mathrm{a}} \approx 1.5 \mathrm{~A}$ $2.2 \mathrm{kV}, \mathrm{V}_{\mathrm{acc}}=500 \mathrm{~V}, \mathrm{I}_{\mathrm{b}} \approx 5 \mathrm{~A}$. Note that in frame (b) the time scale is much longer that frame a, $2 \mu \mathrm{s}$ instead of $0.1 \mu \mathrm{s}$. (two shots are shown); lower frame VRE.HV =

Since it is also expected that an electron cloud-plasma is formed, an accelerating voltage has been added. The emitted current for 1.2 and $2.2 \mathrm{kV}$ driving pulse (corresponding to a field strength of 12 and $20 \mathrm{kV} / \mathrm{cm}$ respectively) and $\mathrm{V}_{\mathrm{acc}}=500 \mathrm{~V}$ of accelerating voltage is shown in fig. 9: the current is one order of magnitude higher than before. However, in the two cases the behaviour is completely different. At the lower exciting voltage, the current amplitude jumps up of a factor five even if the accelerating voltage is only $500 \mathrm{~V}$, the emission starts just when the exciting pulse reaches the maximum (or some nanoseconds later), the waveform has a width of about $500 \mathrm{~ns}$ and shows two peaks as seen in previous fig. 8 .

The high current with low accelerating voltage is consistent with the inference that within the diode there is plasma formation which makes the diode impedance low. Further, the ferroelectric energetic electrons in combination with the plasma form an electron cloud which is ready for the conduction. We notice that the two ferroelectric peaks 400 ns apart which appear without accelerating voltage in fig. 8, are bridged when the accelerating voltage is applied as shown in fig. 9. The plasma, once formed, has its own lifetime. It is about $10 \mu \mathrm{s}$, as shown in frame (b) of fig. 9 (and confirmed also with 4/95/5 sample, see below) when the exciting pulse is well above the threshold of the exciting voltage, the lifetime is, instead, about $0.5 \mu$ s when the exciting voltage is nearby threshold as in frame a of fig. 9. We notice that the two ferroelectric peaks remain evident also with accelerating voltage. The current onset appears at about $50 \mathrm{~ns}$ after the front of the high voltage pulse.

The current waveform changes dramatically when the exciting pulse is high enough, even if the accelerating voltage is kept constant (see fig.9 frame (b)): it has a duration of about $10 \mu \mathrm{s}$, it shows oscillations and the current amplitude reaches a value of $5 \mathrm{~A}$. The impedance of the diode results in only some $\Omega$. Recalling that the drift velocity of a plasma in a field strength of $0.5 \mathrm{kV} / \mathrm{cm}$ can be estimated as a fraction of $\mathrm{cm}$ per $\mu \mathrm{s}$, that waveform and the fact that the diode is almost short circuited are clear indications of plasma formation and its subsequent drift within the gap. 


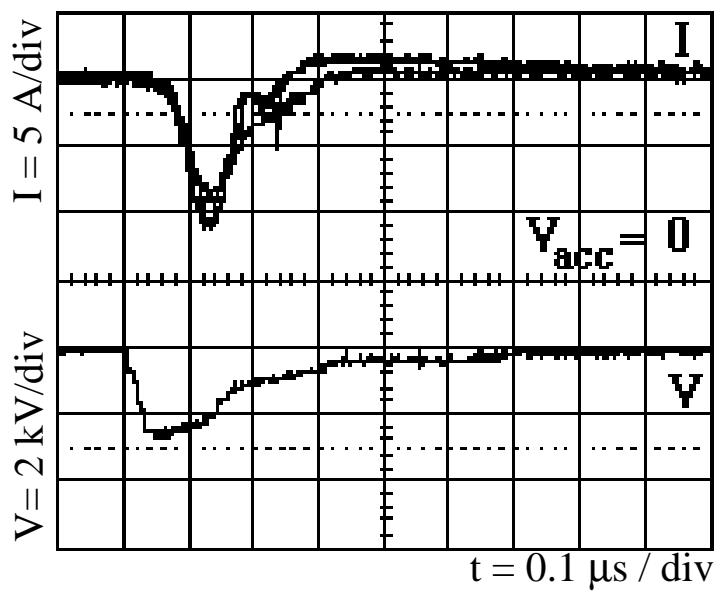

(a)

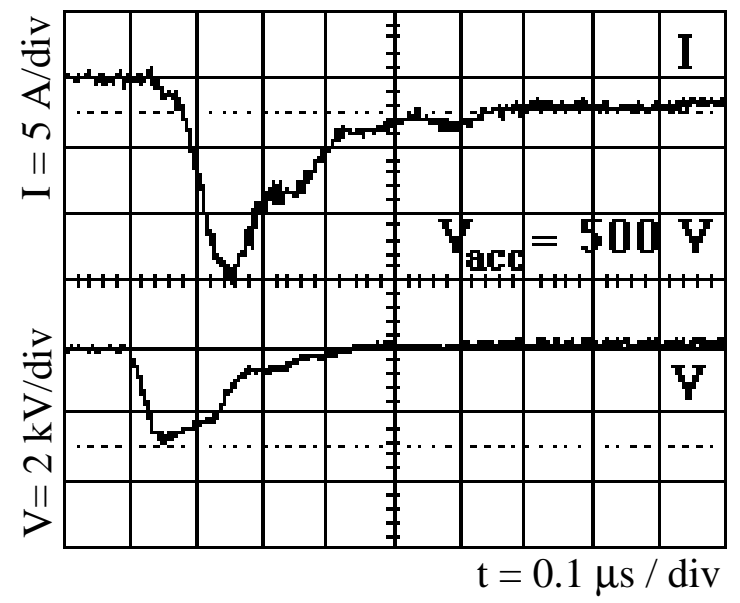

(b)

Fig. 10. Typical waveforms of the current: frame a) $\mathrm{V}_{\text {GE,HV }}$ pulse $=-2.2 \mathrm{kV}$ and $\mathrm{V}_{\mathrm{acc}}=0: \mathrm{I}_{\mathrm{a}} \approx 10 \mathrm{~A}$ (three shots are reported); frame b) as in frame a) but with $\mathrm{V}_{\mathrm{acc}}=500 \mathrm{~V}$.

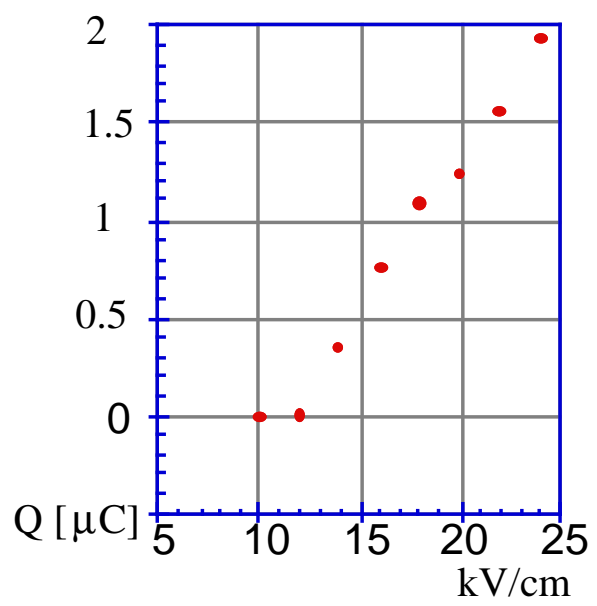

Fig. 11. Diagram of the emitted charge as a function of the amplitude of the switching pulse.

4.2 - Sample PLZT 495/5.

We have tested the emission of the sample 4/95/5. For completeness we have done also tests with 4/94/6 and 2/94/6 and these gave results quite similar to 4/95/5 material. (b) Negative pulse on grid electrode. In this experimental configuration, as said before, the negative HV pulse arriving at the grid excites the ferroelectric crystal and in the meanwhile applies an accelerating voltage of the pulse amplitude. The emitted current waveforms shown in fig. 10 can be compared with those ones of fig. 9 frame (a).

The experimental result with this configuration has mostly the characteristics of previous configuration. The current amplitude is high (because of both plasma formation and accelerating voltage), the current onset is again about $50 \mathrm{~ns}$ after the exciting pulse, the waveform shows two peaks. However, the second peak is small compared with the first one and the current pulse duration is about $150 \mathrm{~ns}$, contrary to the $500 \mathrm{~ns}$ of previous configuration. Adding the accelerating voltage of $500 \mathrm{~V}$, the second peak increases, but the current pulse shape remains substantially stable. The lifetime of the plasma is shorter than the previous case as indicated by the pulse duration.

We repeated the measurements at different values of the temperature up to $100{ }^{\circ} \mathrm{C}$. The variation of emitted current with temperature was almost negligible with this kind of material.

In this experimental configuration of the exciting pulse applied to front electrode, we have measured the emitted charge as a function of the amplitude of the HV pulse. The result is shown in fig. 11.

The relation between the current (the pulse shape is reasonably stable) and the voltage comes out to be linear, after the threshold value. This value results around $8 \mathrm{kV} / \mathrm{cm}$. The resistance of the diode resulted in about $200 \Omega$ (the diode sees $100 \Omega$ towards ground).

Incidentally, we point out that in this experiment, for the fact that the change of the HV pulse brings about simultaneously the change of the spontaneous polarization $\mathrm{P}_{\mathrm{S}}$ of the sample and of the accelerating voltage $\mathrm{V}_{\mathrm{acc}}$, two parameters vary in the same time. 
We wanted to investigate with these samples the same plasma ferroelectric assisted regime. Since the basic idea supporting this regime is that the HV pulse induces a variation of polarization, which in turn creates a repulsing force for the electrons gathered on the surface of the crystal, these samples must operate at high temperature, where they are within the ferroelectric phase. The above mentioned samples had a low unstable emission at room temperature, whilst they emitted regularly once heated at $\mathrm{T}>130^{\circ} \mathrm{C}$.

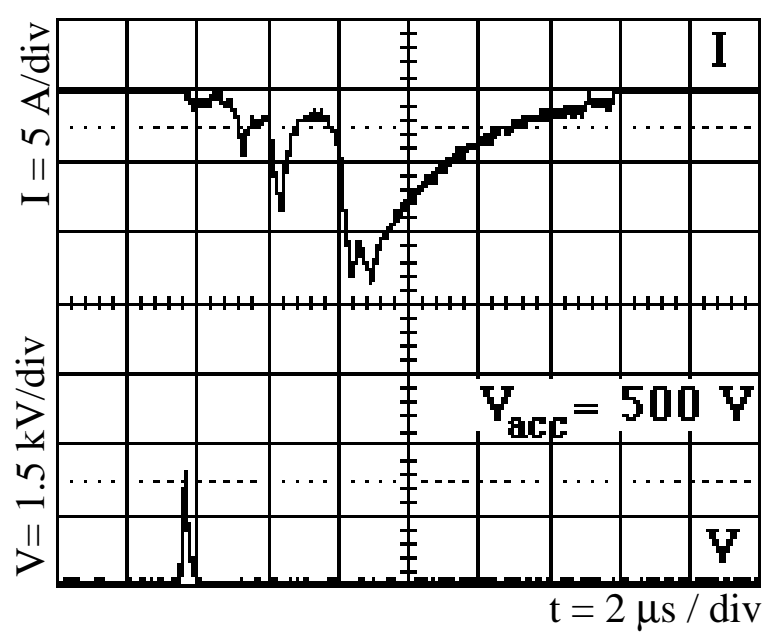

Fig. 12. Typical current waveform $\mathrm{VRE}, \mathrm{HV}=2.5 \mathrm{kV}$ (bottom curve), $\mathrm{V}_{\mathrm{acc}}=500, \mathrm{I} \approx 10 \AA$ (top curve), $\mathrm{T} \approx 180^{\circ} \mathrm{C}$.
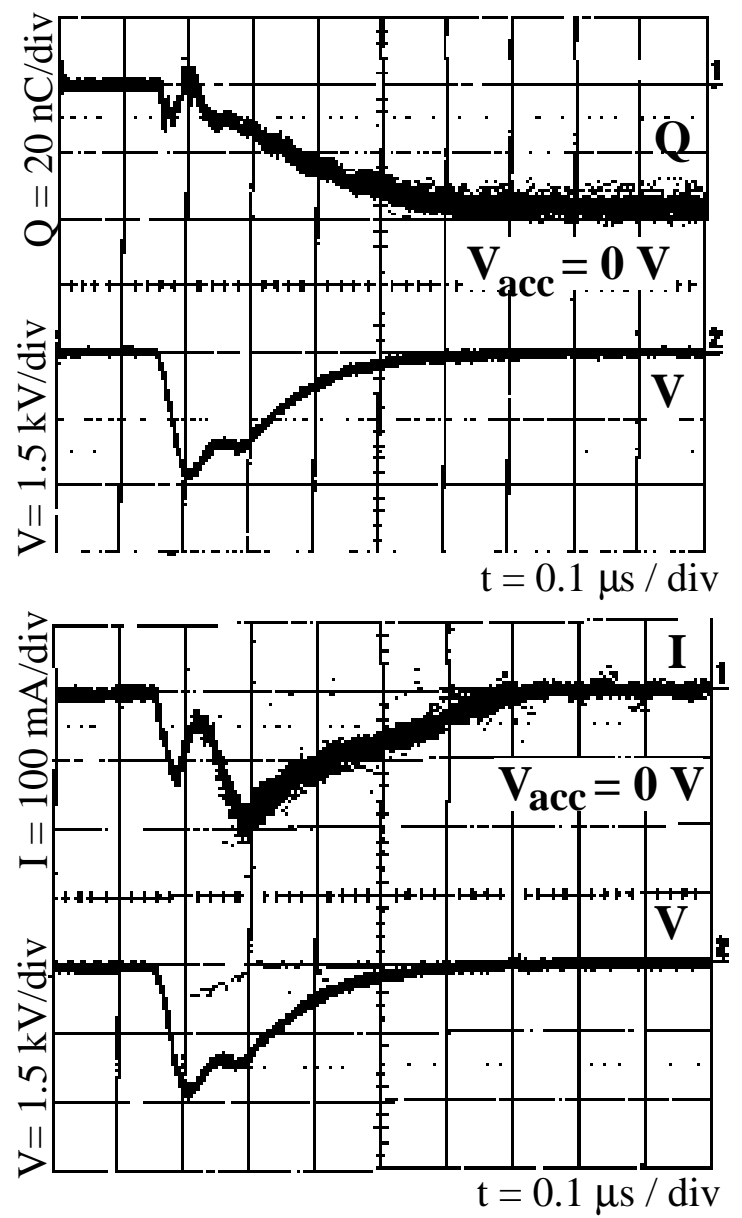

Fig. 13. Typical current waveform (60 shots recorded) $\mathrm{V}_{\mathrm{GE}, \mathrm{HV}}=2.5 \mathrm{kV}$ (bottom curve), $\mathrm{V}_{\mathrm{acc}}=0$, upper frame: current $\mathrm{I} \approx 180 \mathrm{~m} \mathrm{~A}$ (top curve), lower frame charge $\mathrm{Q}=40 \mathrm{nC}$. (a) Positive pulse on rear electrode

Applying a positive pulse on rear electrode, we observe a very small current. This means that the ferroelectric electrons are not enough energetic to counteract the space charge force.

When the accelerating voltage of $500 \mathrm{~V}$ was added, the emission was as strong as for the $8 / 65 / 35$ sample, as shown in fig. 12. The waveform has the typical shape with some oscillations, the value of the current at the peak is $\mathrm{I} \approx 5$ A and its duration is $\tau \approx 10 \mu \mathrm{s}$.

(b) Negative pulse on front electrode and $\mathrm{V}_{\mathrm{acc}}=0$

In fig. 13, the current and charge emitted in this configuration are reported. The emitted current is considerably less than with previous material 8/65/35, $0.2 \mathrm{~A}$ compared with few amps. The shape of the current pulse is different, and its onset is retarded compared with the previous case. It seems that the plasma formation within the one hundred nanosend of the HV pulse duration is week with this material.

It must be pointed out that the first peak on the left is not current, it is the disturbance generated by the capacitive coupling of the Pierce electrode with the front disk collector. In previous figures that small peak was not evident because the high current amplitude required a different scale of the oscilloscope.

We have measured the emitted charge versus the temperature and versus the exciting voltage strength at different temperatures. The curves are reported in fig. 14. There is a threshold around $130^{\circ} \mathrm{C}$, which corresponds approximately to the antiferro- 
ferroelectric phase transition temperature: the curves below this temperature, shown in frame b, are substantially stable near zero. Starting from that value of temperature the rate of the emission with field strength increases very fast.

(a)
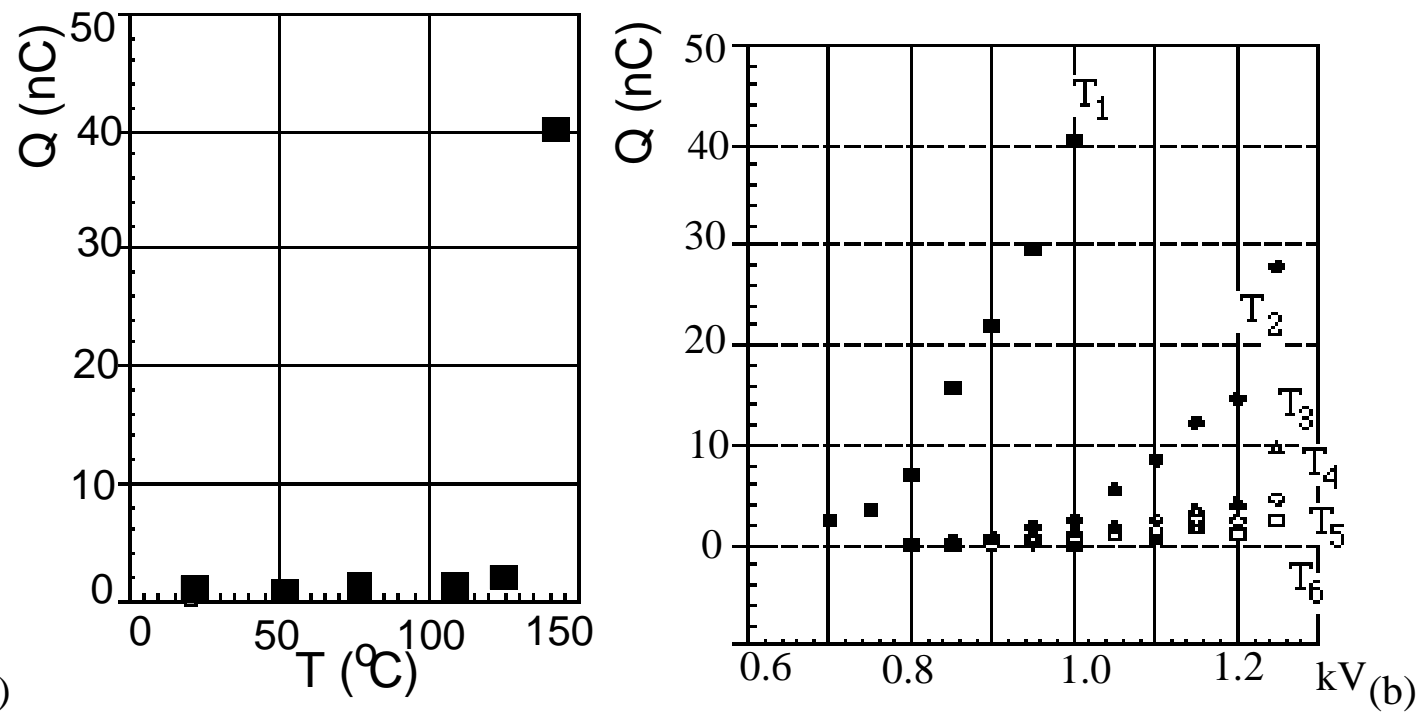

Fig. 14. Frame (a): Emitted charge versus temperature with $\mathrm{V}_{\mathrm{HV}}, \mathrm{GE}=2.8 \mathrm{kV}$; frame (b): Emitted charge versus excitation voltage for different temperatures: $\mathrm{T}_{1}=142^{\circ} \mathrm{C} \quad \mathrm{T}_{2}=125^{\circ} \mathrm{C} \quad \mathrm{T}_{3}=110^{\circ} \mathrm{C} \quad \mathrm{T}_{4}=75^{\circ} \mathrm{C}$ $\mathrm{T}_{5}=50^{\circ} \mathrm{C} \quad \mathrm{T}_{6}=20^{\circ} \mathrm{C}$.

\section{5- Conclusions}

Ferroelectric solid solutions PLZT of 8/65/35 and 4/95/5 compositions have been tested as electron emitters in a diode with a low accelerating field and also without an accelerating field. The excitation has been done either with a positive electric pulse of $200 \mathrm{~ns}$ duration on the rear side of the ferroelectric disk, or a negative pulse on the front (grid) side. The disks have emitted in both cases with and without accelerating field.

The $300 \mathrm{~mA}$ emitted by the 8/65/35 cathode without accelerating field (positive pulse on the rear side), establishes that a fraction of the emitted electrons are energetic, since a space charge force opposes their emission. The waveform shows almost constantly two peaks. After the switching off of the high voltage pulse, there is a kind of polarization oscillation which leads to two (and sometime more) ferroelectric peaks.

The 10-15 A current obtained either adding an accelerating voltage in case of rear positive excitation, or pulsing the front side with $2 \mathrm{kV}$ pulse tells that (a) most of electrons are not energetic and $b$ ) the diode impedance is only about $200 \Omega$, hence plasma and/or an electron cloud is formed in front of the emitting cathode. Also, in this configuration the waveform shows two peaks.

When the positive excitation is high enough and an accelerating voltage of only $500 \mathrm{~V}$ has been applied through the $10 \mathrm{~mm}$ diode gap, the emitted current pulse was very long, about $10 \mu \mathrm{s}$, had some oscillations and the peak amplitude was near $5 \mathrm{~A}$. This current with that voltage means that the diode impedance was only some $\Omega$ (the applied resistance towards ground was $100 \Omega$ ).

The 8/65/35 material operates at room temperature, while 4/95/5 worked at $\mathrm{T}>130^{\circ} \mathrm{C}$. The latter was much less efficient that the former in this particular excitation method.

The experimental results show clearly that, in the configuration with excitation field directed from the rear side towards the grid side of the sample and no accelerating field through the diode gap, the collected electrons are those acted upon by the switching polarization. In short, the exciting voltage applied to the cathode switches a spontaneous polarization, which creates a deep sink for external electrons; when the external electric field is switched off, those electrons remain uncompensated and are, then, pushed away from the crystal surface. The electrons are, in fact, energetic. When an accelerating field is 
applied, the collected electrons seem to come mostly from a plasma, which forms in front of the cathode because of the field emission from the metal stripes deposited over the crystal. This current is, therefore, related to a different physical phenomenon compared with previous one. The so-called ferroelectric current pulse appears about $50 \mathrm{~ns}$ after the end of the switching pulse, while the plasma formation starts with the front end of the pulse.

We remark that the plasma formation within the diode is harmful for a fast repetition rate of a possible electron source.

\section{REFERENCES}

[1] H. Gundel, J. Handerek, H. Riege, E.J.N. Wilson and K. Zioutas, Ferroelectrics 100, 1, 1989.

[2] H. Gundel, J. Handerek, H. Riege, E.J.N. Wilson and K. Zioutas, Ferroelectrics 109, 137, 1990.

[3] H. Gundel, J. Handerek, H. Riege, JAP 69,975,1991.

[4] K. Geissler, H. Gundel, J. Handerek, H. Riege, Appl. Phys. Lett. 56, 895, 1990.

[5] K. Geissler, H. Gundel, J. Handerek, H. Riege, Appl. Phys. Lett. 56, 895, 1990.

[6] K. Geissler, J. Handerek, A. Meineke, H. Riege, K. Schmidt, Phys. Lett. A176, 387, 1993.

[7] A.S. Airapetov, I.I. Ivanchink, A.N.Lebedev, I.V. Levshin, and N.A. Tikhomirova., Sov. Phys. Dokl. 35,267, 1990.

[8] G. Rosenman, Ferroelectrics 126, 305, 1992.

[9] J. Asano, T. Imai, M. Okuyama and Y. Hamakawa, Jpn., J. Appl. Phys. 31, 3098, 1992.

[10] K. Bierdrzycki, R. Le Bihan, Ferroelectrics 124, 1201, 1992.

[11] J. D. Ivers, L. Schächer, J. A. Nation, G. S. Kerslick and R. Advani, J. Appl. Phys. 79, 2667, 1993.

[12] E. Zhang Conf. Proc. of the 1993 PAC, Washington D.C., May 1993.

[13] S. E. Sampayan, G. J. Caporaso, C. L. Holmes, E. J. Lauer, D. Prosnitz, D.O. Trimble, G. A. Westenskow, Nucl. Instr. Meth. Phys. Res. A 340, 90, 1994.

[14] D. Viehland, S.J. Jang, L. E. Cross, M. Wutting, J. Appl. Phys. 68, 2916, 1990.

[15] S. H. Haertling, Ceramic Material for Electronic, pag 129, Bacharian, New York 1991.

[16] J. Handerk, Z. Ujma, C. Carabatos-Nèdelec, G. E. Kugel, D. Dmytrov, I. ElHarrad, J. Appl. Phys. 73, 367, 1993.

[17] L. Schachter, J.D. Ivers, J.A. Nation, G.S. Kerslick, J. Appl. Phys. 73, 8097, 1993.

[18] I Boscolo et al. Appl. Phys. B Springer Verlag 50, 71, 1990.

[19] G. R. Brewer, in Focusing of charged particles, Vol. II, ed. By A. Septier, Accademic Press, New York, pp 23-72. 